\title{
KiWi - A Platform for Semantic Social Software (Demonstration)
}

\author{
Sebastian Schaffert, Julia Eder, Szaby Grünwald, Thomas Kurz, \\ and Mihai Radulescu \\ Salzburg Research Forschungsgesellschaft \\ Jakob Haringer Str. 5/II, A-5020 Salzburg, Austria \\ firstname.lastname@salzburgresearch.at
}

\begin{abstract}
Semantic Wikis have demonstrated the power of combining Wikis with Semantic Web technology. The KiWi system goes beyond Semantic Wikis by providing a flexible and adaptable platform for building different kinds of Social Semantic Software, powered by Semantic Web technology. While the KiWi project itself is primarily focussed on the knowledge management domain, this demonstration shows how KiWi aspects like the Wiki Principles and Content Versatility can be used to build completely different kinds of Social Software applications. The first application we show is an "ordinary" Semantic Wiki system preloaded with content from a online news site. The second application called TagIT is a map-based system where locations and routes on a map can be "tagged" by users with textual descriptions, SKOS categories, and multimedia material. Both applicatons are built on top of the same KiWi platform and actually share the same content.
\end{abstract}

\section{Motivation: From Semantic Wikis to KiWi}

Semantic Wikis have been under investigation in the research community since 2005 (see e.g. [12]). Although there are many different systems with many different properties, a common trait between all Semantic Wikis is that they aim to combine ordinary Wiki content and technology with Semantic Web technologies, either in order to provide better wikis ("Semantic Web for Wikis") or to ease the creation of Semantic Web data ("Wikis for the Semantic Web") 3. Many also see Semantic Wikis as the "Semantic Web in a Nutshell", because - like Wikis show similar traits to the Web as a whole - Semantic Wikis share many properties and also problems with the envisioned Semantic Web.

The EU-funded project KiWi ("Knowledge in a Wiki") takes the Semantic Wiki approach to the next level by providing a platform that allows to build many different kinds of Social Semantic Software, based on the conviction that most social software follows the "Wiki Principles". By "Wiki Principles", we mean that the term "Wiki" does not refer to technology alone. It is more a new philosophy of working with Web content influenced by ideas in the OpenSource community. These principles have revolutionised the way we work with content in the (Social) Web: 
- Wikis allow anyone to edit: The core principle is that there are no access restrictions or strict hierarchies on the content of a wiki. Anyone can easily contribute his or her own knowledge, his or her own ideas, and his or her own content.

- Wikis are easy to use: Anyone who is sufficiently familiar with the basic functionalities of word processing software (write, delete, save) has all the skills required to edit, correct and expand a wiki.

- Wiki content is linkable: By allowing users to create links between words and as such between concepts, wikis also allow for the creation of semantic relations, i.e. of meaning.

- Wikis support versioning: Never does information disappear on a wiki. If a page is edited, the previous version is still stored somewhere. This has an important psychological effect as it takes away the wiki writer's block: the fear that something might get lost through editing.

- Wikis support all media: Wikis are web-based. So whichever type of content you have, be it text, images, audio, spreadsheets, documents - anything that can be displayed in a web browser can be displayed in a wiki. And even if a file cannot be displayed in the browser itself, it can still be downloaded.

This same philosophy is underlying not only Wikis (technically-speaking), but also a large array of other Social Software applications: e.g., a weblog or social networking platform can be seen as just a different user interface (and different way of using), but is otherwise very similar concerning the underlying principles and also technology.

\section{Content Versatility: Same Content, Different Views}

Following this line of argumentation, it is close at hand to build generic platforms for developing different kinds of Social Software. And indeed, there are several products already available that aim to deliver a platform that allows to combine wikis, weblogs, social networking, etc. Such platforms are for example Clearspace Community 1 from Jive Software, Community Server 2 from telligent, and Liferay Social Office 3 from Liferay. However, all these systems only provide integration on the user interface level and still see wiki articles, blog posts, etc. as separate kinds of content that is visualised in a specific way.

KiWi follows a completely different approach which we call "Content Versatility". The underlying principle is that every piece of information is a combination of human-readable content and associated meta-data, and that the same piece of information can be presented to the user in many different forms, even in parallel: as a wiki page, as a blog post, as a comment to a blog, as a photo, or even in a bubble in a map-based application. The decision how the information is displayed is taken based on the context of the content and the user (e.g. metadata, user preferences, different applications).

\footnotetext{
1 http://www.jivesoftware.com/products/clearspace-community

2 http://communityserver.com/

3 http://www.liferay.com/web/guest/products/social_office
} 

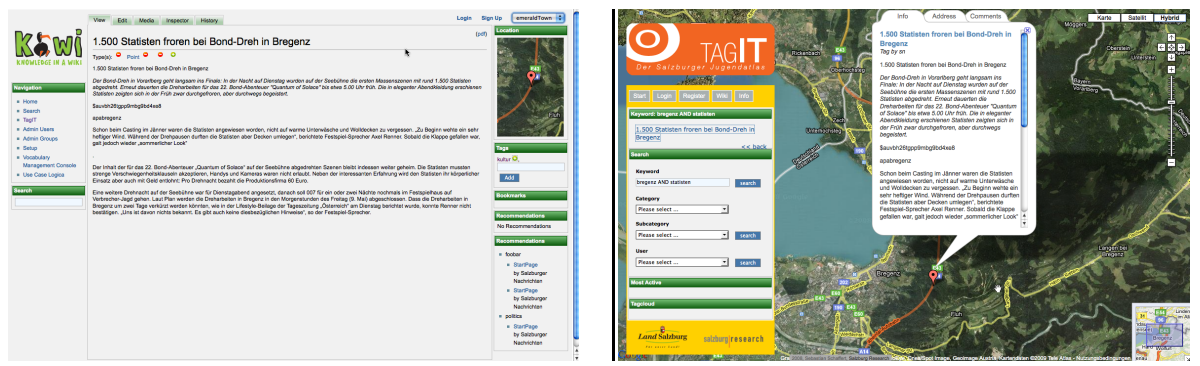

Fig. 1. KiWi Applications: the same content item in the KiWi Wiki on the left, and in TagIT on the right

In KiWi, we call the smallest piece of information a "content item". A content item is identified by a URI and consists of human-readable content in the form of XHTML and associated meta-data in the form of RDF. The KiWi core system provides means to store, update, search, and query content items, and offers automatic versioning of all updates (content and meta-data). Whereas core properties of a content item (like the content, the author, and the creation date) are persisted in a relational database, all other properties can be flexibly defined using RDF properties or relations. The URI of a content item is generated in such a way that it is possible to make a KiWi system part of the Linked Open Data cloud [4. In the following, we present two KiWi applications that share the same content but have completely different ways of presenting it.

The KiWi Wiki. The primary and most generic interface to the KiWi system is a Semantic Wiki. The layout and functionality (Figure1left) of the KiWi Wiki is inspired by its predecessor IkeWiki[5]: the browser view is divided into three columns where the left column provides navigation functionality and access to tools, the center column contains the main (human-readable) wiki content, and the right column contains dynamic widgets that display additional information about the content item based on its metadata (e.g. a map or incoming and outgoing links). The center column by default displays the content of the content item and the user can switch to editing, to annotation, and to history by using tags displayed on top of the content. The KiWi Wiki interface can be used in the same way as IkeWiki, with some additional features.

TagIT. TagIT is an application originally developed in a separate project with the goal to create the "Youth Atlas of Salzburg", which has now been ported to the KiWi platform (Figure 1 on the right). In TagIT, users browse through a map (based on Google Maps) where they can "tag" locations with descriptions and provide interesting information about them, e.g. cafés, bars, sports parks, hiking tours, etc. Such tags can be associated with categories from a SKOS thesaurus and with additional multimedia data like photos or videos. Other users can then browse or search for interesting locations using the same interface. In addition to the web-based platform, TagIT also offers a mobile client that can run on a GPSenabled mobile phone. When visiting an interesting location, users can then start 
the TagIT application, take a picture of the location, add a short description and directly upload the "tag" using UMTS. The tag is automatically geolocated and immediately available for other users.

Although quite different on the user interface and in the way it is used, TagIT actually closely follows the wiki principles: everyone can add and edit tags, the system is extremely easy to use, tags can be linked, tags are versioned, and different kinds of content are supported. On top of $\mathrm{KiWi}$, tags are realised as content items and can thus be displayed in both the TagIT user interface and the previously described KiWi Wiki (in which case a small map widget is displayed in the right column showing the position).

The two user interfaces are currently implemented as separate applications on top of the KiWi core system. In further phases of the project, the KiWi system will also provide reasoning and personalisation support that will allow to flexibly decide which presentation of a content item to choose for a given user and context.

\section{Demo Outline}

The demonstration we aim to give at ESWC will first show the core features of the KiWi system, namely the use and features of the KiWi wiki and the TagIT system and their semantic properties. A particular focus will be on the demonstration of the "Content Versatility", as it is a fundamental concept of KiWi and demonstrates the power and flexibility of using Semantic Web technologies for Social Software applications.

For the purpose of demonstrating the KiWi system in a real-world scenario, we will preload the system with a selection of news articles with geoinformation from our partner, Salzburger Nachrichten. We will then show how such articles can be browsed, searched, and edited in the KiWi wiki and in the TagIT system, and we will demonstrate that both user interfaces are really just different views on the same content. To demonstrate the flexibility of the system, we will also show how the mobile client can be used to add new geo-located content to the system and browse the system for interesting locations. Depending on the state of the KiWi system at the time of the conference, we furthermore aim to show advanced features like personalisation, and we might be able to demonstrate the integration of additional Salzburger Nachrichten content beyond news articles.

\section{Perspectives and Conclusion}

$\mathrm{KiWi}$ is an ongoing research project of which we have only demonstrated the first results. Many more salient aspects are still to come. Particularly, KiWi will be extended with "enabling technologies" in the following areas:

- Reasoning and Reason Maintenance: In this area, the KiWi system will be extended with a custom, rule-based querying and reasoning component where advanced users will be able to add custom inference rules to the 
knowledge base; reasoning will be based on content as well as meta-data 6]. In addition, there will be a reason maintenance component that allows users to get explanations why a certain information has been inferred; reason maintenance is also beneficial for update performance and might even allow for different users having different rule sets.

- Information Extraction: The KiWi system will furthermore provide a component that semi-automatically extracts meta-data from the content that is stored in the knowledge base. Information Extraction will be performed in interaction with the user to minimise the number of errors.

- Personalisation: Based on the meta-data for a content item, a user model, and the rule-based reasoning, KiWi will also offer a personaliation component that allows to further customise the presentation of a content item. The personalisation component will further demonstrate the flexibility of the Content Versatility approach taken by KiWi.

TagIT is now further developed as part of the Salzburg NewMediaLab project "Future Content Platforms" together with our partner Salzburger Nachrichten. The goal is to integrate in the same interface not only wiki content and TagIT locations but also news articles, blog posts, and small advertisements from our partner. For the purpose of the demonstration, we have already imported 20.000 online news articles, but the system is designed to scale to hundreds of thousands.

Acknowledgement. This research has been partly funded by Salzburg New Media Lab and by the the European Commission within the 7th Framework Programme project KiWi - Knowledge in a Wiki (No. 211932). The latest KiWi source code is available as Open Source at the KiWi website http://www.kiwi-project.eu. The demonstration system is published from time to time at http://showcase.kiwi-project.eu

\section{References}

1. Völkel, M., Schaffert, S. (eds.): 1st Workshop "From Wiki to Semantics" (SemWiki 2006) - colocated with ESWC 2006, Budva, Montenegro (2006)

2. Lange, C., Schaffert, S., Skaf-Molli, H., Völkel, M. (eds.): 3rd Workshop "From Wiki to Semantics" (SemWiki 2008) - colocated with ESWC 2008, Tenerife, Spain (2008)

3. Schaffert, S.: Semantic Social Software: Semantically Enabled Social Software or Socially Enabled Semantic Web? In: Semantics 2006, Vienna, Austria (2006)

4. Bizer, C., Heath, T., Ayers, D., Raimond, Y.: Interlinking Open Data on the Web. In: Franconi, E., Kifer, M., May, W. (eds.) ESWC 2007. LNCS, vol. 4519. Springer, Heidelberg (2007)

5. Schaffert, S., Westenthaler, R., Gruber, A.: IkeWiki: A User-Friendly Semantic Wiki. In: Sure, Y., Domingue, J. (eds.) ESWC 2006. LNCS, vol. 4011. Springer, Heidelberg (2006)

6. Bry, F., Kotowski, J.: Towards reasoning and explanations for social tagging. In: Proceedings of 3rd International Workshop on Explanation-aware Computing, Patras, Greece, July 21-22 (2008) 